\title{
75 lecciones aprendidas en programas de alfabetización informacional en universidades iberoamericanas
}

\author{
Alejandro Uribe Tirado*, María Pinto** \\ *Escuela Interamericana de Bibliotecología, Universidad de Antioquia, Medellín-Colombia. \\ Correo-e: auribe.bibliotecologia.udea@gmail.com, auribe@correo.ugr.es \\ **Departamento de Información y Comunicación. Universidad de Granada, Granada-España. \\ Correo-e: mpinto@ugr.es, mariapinto@mariapinto.es
}

Recibido: 29-08-2013; 2a version: 25-11-2013; 3a version: 14-01-2014; Aceptado: 27-01-2014.

Cómo citar este artículo/Citation: Uribe Tirado, A.; Pinto, M. (2014). 75 lecciones aprendidas en programas de alfabetización informacional en universidades iberoamericanas. Revista Española de Documentación Científica, 37(3):e057. doi: http://dx.doi. org/10.3989/redc.2014.3.1118

Resumen: El objetivo de este artículo es ofrecer una sistematización y organización de las lecciones aprendidas desde los programas más representativos de Alfabetización Informacional -ALFIN- en universidades iberoamericanas. Tras la aplicación de un modelo de captura de lecciones aprendidas basado en la triangulación de diferentes fuentes de información y métodos de investigación (análisis de la literatura y sitios web, entrevistas, encuestas, etc.), se ofrece como resultado las 75 lecciones aprendidas identificadas, categorizadas según cuatro grandes componentes, para que a modo de guía puedan ser utilizadas por las universidades iberoamericanas iniciadas y/o principiantes en el diseño de sus programas de formación en Alfabetización Informacional.

Palabras clave: Alfabetización Informacional; competencias informacionales; lecciones aprendidas; gestión del conocimiento para el aprendizaje; universidades; Iberoamérica.

\section{5 lessons learned from information literacy programs in Ibero-American universities}

Abstract: The aim of this article is to provide a systemized and organized presentation of the lessons learned from the most representative programs of Information Literacy in Ibero-American universities. A model was applied to capture lessons learned based on the triangulation of different sources of information and research methods (analysis of literature and websites, interviews, surveys, etc...). This resulted in the identification of 75 lessons learned, categorized by four main components, that can serve as a guide to be used by Ibero-American universities that have already initiated the design and implementation of training programs in Information Literacy, as well as those just beginning this process.

Keywords: Information literacy; information competencies; lessons learned; knowledge management for learning; university; Ibero-america.

Copyright: ( 2014 CSIC. Este es un artículo de acceso abierto distribuido bajo los términos de la licencia Creative Commons Attribution-Non Commercial (by-nc) Spain 3.0. 


\section{INTRODUCCIÓN}

Esta investigación supone una continuación de trabajos previos sobre alfabetización informacional (Uribe-Tirado, 2004; Pinto y Doucet, 2007; UribeTirado, 2008; Pinto y otros, 2008; Uribe-Tirado y otros, 2008; Pinto, 2010; Pinto y Uribe-Tirado, 2011; Pinto y otros, 2013) relacionados con la identificación de dos realidades en el contexto de las universidades y bibliotecas iberoamericanas.

En primer lugar, nos referimos a la necesidad de los estudiantes universitarios iberoamericanos en cuanto a su formación en competencias informacionales. A lo largo de los últimos años, distintas investigaciones muestran que los estudiantes "nativos digitales" al llegar a la universidad e incluso durante su estancia, tienen unas competencias informacionales moderadas cuando no existe un programa de ALFIN estructurado. Desafortunadamente, en los curricula de los niveles escolares de primaria y secundaria no ha estado presente esta formación informacional, ni tampoco ha sido fomentada desde las bibliotecas escolares o públicas. Esta circunstancia se diferencia del nivel universitario, donde se observan algunos desarrollos destacados aunque puntuales (Pinto y Uribe-Tirado, 2012).

En segundo lugar, pese a que la ALFIN no tiene el mismo desarrollo en el contexto de todas las universidades e instituciones de educación superior (IES), son las bibliotecas universitarias las que más han avanzado. A veces esos casos no son muy conocidos, o cuando se conocen sólo destacan los resultados, cursos, etc., pero no los procesos de diseño de los programas de formación y su impacto en la universidad. De ahí que resulte de interés conocer esos procesos y las lecciones aprendidas, que servirían de referente para la mejora de los programas de formación propios o de contextos semejantes.

En este sentido, la hipótesis que guía este trabajo, expresa que la formación en competencias informacionales, mediante el uso de diferentes estrategias didácticas, ambientes de aprendizaje y recursos de información, permitirá a los estudiantes universitarios mayores posibilidades de adquisición de conocimientos, habilidades y actitudes para una adecuada gestión de la información y el conocimiento, logrando un mejor desempeño académico, científico y ciudadano durante su vida universitaria y profesional. El objetivo de este trabajo es identificar, analizar, organizar y compartir las lecciones aprendidas en los programas de formación en ALFIN llevados a cabo en las universidades iberoamericanas desde diferentes contextos, con el propósito de generar mejores propuestas, además de redes de apoyo y de evaluación conjunta, considerando las potencialidades que supone compartir idioma y prácticas sociales y culturales.

\section{INTERRELACIÓN ENTRE LECCIONES APRENDIDAS Y ALFIN}

En los últimos años uno de los conceptos más empleados en los ámbitos organizacionales y edu- cativos es el de Lecciones Aprendidas (LA). La razón para este uso frecuente se debe al desarrollo en las últimas tres décadas de dos corrientes teórico-prácticas que desde el ámbito empresarial se han ido desplegando y adaptando a las exigencias de la sociedad actual: Aprendizaje Organizacional -Organizational Learning- y Conocimiento Organizacional -Organizational Knowledge-. Estas corrientes no son un producto y resultado aislado del cambio generado por las tecnologías de la información y la comunicación -TIC-, sino más bien la consecuencia de la influencia de éstas en las formas de producción de bienes y servicios. Así se da paso a una generación donde la "riqueza" más orientada al conocimiento y donde el aprendizaje permanente (personal y organizacional) y el trabajo en red (de personas y sistemas) son el eje medular.

Este cambio de orientación, enmarcado en las TIC, ha dado origen al concepto de Sociedad de la Información, Sociedad del Conocimiento, Sociedad de la Información y del Conocimiento, etc., según la posición que se asuma en función de determinados postulados.

El desarrollo de estas dos corrientes muestra que son complementarias (Easterby-Smith y Lyles, 2003) ya que, como en todos los campos, teoría y práctica se retroalimentan mutuamente. Este fenómeno retro-alimentador se reproduce en lo que a procesos y contenidos se refiere. Esto implica, en el contexto de las LA, la necesidad de considerar qué se entiende por Aprendizaje Organizacional y Conocimiento Organizacional, por Organización que aprende y Organización que gestiona sus conocimientos (Gestión del conocimiento), y las interrelaciones de estos conceptos.

No es de extrañar entonces que las LA, como concepto y práctica, hayan tenido presencia directa e indirecta en ambas corrientes y en dichos cuatro conceptos, pues implican la convivencia de procesos y contenidos (resultados), un ambiente de trabajo y de análisis propicio para identificar y compartir aprendizajes, y el uso de diferentes estrategias y herramientas que facilitan la creación, captura, organización, transferencia y/o recuperación de los conocimientos concretos de las personas que integran la organización, y la organización misma.

Por consiguiente, desde una perspectiva amplia e integradora, entendemos por lecciones aprendidas $(L A)$ :

Los conocimientos tácitos y explícitos que genera un individuo, grupo u organización a partir de una experiencia significativa (positiva o negativa) en el desarrollo de una actividad, que pueden ser registrados y compartidos mediante diferentes espacios, estrategias y herramientas, para que otros individuos o grupos de la misma organización, o de otras organizaciones que desarrollen prácticas semejantes, puedan aprender de esos conocimientos basados en la experiencia y generar acciones de cambio. Acciones 
de cambio, con las adaptaciones contextuales necesarias para que sean pertinentes, y así, poder obtener procesos o resultados semejantes, reforzar prácticas positivas que ya se están realizando, o evitar las negativas, buscando con todo ello la mejora continua del quehacer personal, profesional y organizacional.

Esta definición propia implica que las LA son tanto un proceso-resultado como un medio-fin, que ayuda a la generación de espacios y posibilidades de aprendizaje permanente a un individuo, grupo u organización específica (o en una temática que integra diferentes organizaciones, como es el caso de ALFIN en las universidades iberoamericanas). De este modo se contribuye al crecimiento del Conocimiento Organizacional, al ser éste una estrategia y herramienta útil para la creación, captura, estructuración, difusión, adquisición y/o aplicación de conocimientos.

Como afirma Milton (2010): "If an organization can draw lessons from experience, can eliminate repeating mistakes, and can reproduce success, then the result should be a continuous improvement in performance... If people in team deliberately focus on learning from their own activity, they can accelerate, or steepen, their learning curve. This creates value, as they can reach lower cost or higher efficiencies faster than if they were learning naturally".

Por consiguiente, si facilitamos el conocimiento de lo que otros han desarrollado con importantes resultados (Benchmarking) y las buenas-mejores prácticas en este campo, se logrará un mayor nivel de conocimiento gracias a la capitalización de las experiencias y al valor de compartir el conocimiento.

\section{MÉTODOS E INSTRUMENTOS PARA IDENTIFI- CAR LECCIONES APRENDIDAS EN PROGRAMAS DE ALFIN: BASES PARA EL MODELO}

En consonancia con la definición propuesta de $\mathrm{LA}$, donde un aspecto fundamental son los conocimientos tácitos y explícitos, así como su gestión, presentamos los diferentes métodos e instrumentos que hemos utilizado. En este punto es importante indicar que, acorde con la metodología de investigación holística que este trabajo asume, se ha realizado una triangulación de métodos cualitativo-cuantitativo que integra las diferentes fases y estrategias, proporcionando una completa localización y recuperación de la información sobre los programas de ALFIN y de sus correspondientes LA. En su aplicación inicial, estos métodos e instrumentos forman parte de un conjunto de herramientas para la captura y la identificación de las lecciones aprendidas (reales y potenciales) desde el paradigma de la gestión del conocimiento ${ }^{1}$. Tras ser validados con una prueba piloto aplicada inicialmente para los programas de ALFIN en universidades de Colombia y Cuba ${ }^{2}$, se emplearon estos cuatro métodos:

\section{Explícito - Documental}

Este método se basa en la búsqueda de evidencias documentales a partir de la literatura científica sobre el tema. Se localizaron numerosos documentos (artículos, ponencias, capítulos de libro, presentaciones, videos, audios, etc.) que reflejan los avances en ALFIN y las formas de incorporación en las universidades iberoamericanas (bien como un todo, o desde la labor de un sistema de bibliotecas, una biblioteca, una unidad académica, etc.). También se recogen la identificación de casos, de programas de ALFIN y de algunas LA.

En este proceso de búsqueda de evidencias documentales se ha empleado preferentemente la técnica de análisis documental de contenido. Por un lado, se han identificado publicaciones internacionales específicas que sistematizan las LA y las buenas prácticas, sirviendo de referente para establecer las categorías de análisis y su codificación inicial. Por otra parte, se ha realizado un análisis documental desde el contexto iberoamericano, para identificar contenidos de orientación teórica y práctica en el desarrollo específico de ALFIN ${ }^{3}$, destacando las universidades que aportan casos concretos. A partir de ambos análisis conceptuales, se ha procedido a la identificación de las LA de una manera estructurada y codificada.

\section{Explícito - Web}

Considerando el enfoque mixto de la investigación basado en la triangulación de datos y métodos, hemos podido observar que aunque la información aportada por el método Explícito-Documental es valiosa, resulta insuficiente para los objetivos marcados. Pese a que la mayoría de los trabajos (Pinto, Uribe-Tirado, Gómez Díaz y Cordón, 2011) proceden del área de información-documentación, se constata que no hay arraigada entre los bibliotecarios iberoamericanos una cultura de publicación y difusión de los resultados.

Por consiguiente, para evitar la ausencia de aportaciones relevantes, se hizo un análisis explícito y directo buscando otros casos significativos que mostraran los avances en este tema y sus respectivas lecciones. De ahí que se procediera a un análisis cuantitativo de los sitios Web de las bibliotecas universitarias iberoamericanas, para identificar cuáles estaban trabajando en ALFIN y destacar sus procesos de formación.

\section{Tácito - Entrevistas}

Teniendo en cuenta los métodos anteriores, somos conscientes que un proceso de captura de las LA implica también un importante trabajo desde un enfoque cualitativo. Se observa que, a menudo, los coordinadores $\mathrm{y} / \mathrm{o}$ formadores de programas ALFIN en Iberoamérica -a pesar de realizar algunos trabajos destacados-, no difunden frecuentemente esas lecciones, como ya lo hemos mencionado. Por tanto éstas permanecen tácitas, bien porque no se han publicado, o bien porque dichos formadores no 
disponen de tiempo para hacer una reflexión crítica que permita difundir lo que suponen esas LA.

Para explicitar esta información implícita, también resultan de utilidad las entrevistas a coordinadores y/o formadores de programas de ALFIN en universidades iberoamericanas. Se empleó la metodología cualitativa, incorporando las aportaciones de la teoría fundamentada (Strauss y Corbin, 2002; Carvalho Dantas y otros, 2009) y considerando la propuesta de Wheeldon y Ahlberg (2012) de cuantificar lo cualitativo y calificar lo cuantitativo: "All quantitative data are based on qualitative judgment; all qualitative data can be described numerically".

\section{Tácito/Explícito - Encuestas}

En relación con el objetivo de buscar directamente la captura de esas LA desde la reflexión de los coordinadores y/o formadores de estos programas en las universidades iberoamericanas, y ante la imposibilidad de entrevistar a todos los responsables identificados, se decidió aplicar la encuesta a una muestra representativa. De esta forma, se pretende capturar de manera indirecta su visión y sus LA conforme a las categorías establecidas en la matriz de recogida de datos (figura 1 , parte inferior).
La encuesta se diseñó de manera online utilizando la aplicación SurveyMonkey, se habilitó en versión bilingüe (español y portugués) y se dividió en dos partes. Mientras la primera recoge los datos básicos para identificar al coordinador y/o formador de ALFIN, la universidad de procedencia y su experiencia en esta temática, la segunda parte se centra en la información específica sobre las LA.

\section{MODELO DE CAPTURA DE LECCIONES APRENDIDAS}

El proceso descrito con anterioridad permite la propuesta de un modelo de captura de las LA que de forma esquemática y sistémica incluye los componentes metodológicos y las interrelaciones entre los conceptos claves (fig. 2). A partir de este modelo validado en la prueba piloto, se diseña una metodología de trabajo viable que permita incluir todos los 22 países iberoamericanos. Desde la pluriculturalidad y diversidad de este colectivo iberoamericano, se pretende construir una visión regional de ALFIN con el propósito de mejorar el conocimiento, apoyo, aprendizaje, crecimiento y maduración mutuos. Ello contribuiría también a una mejora de las aportaciones académicas en el contexto internacional.

Figura 1. Modelo identificación de lecciones aprendidas en programas de ALFIN en universidades iberoamericanas

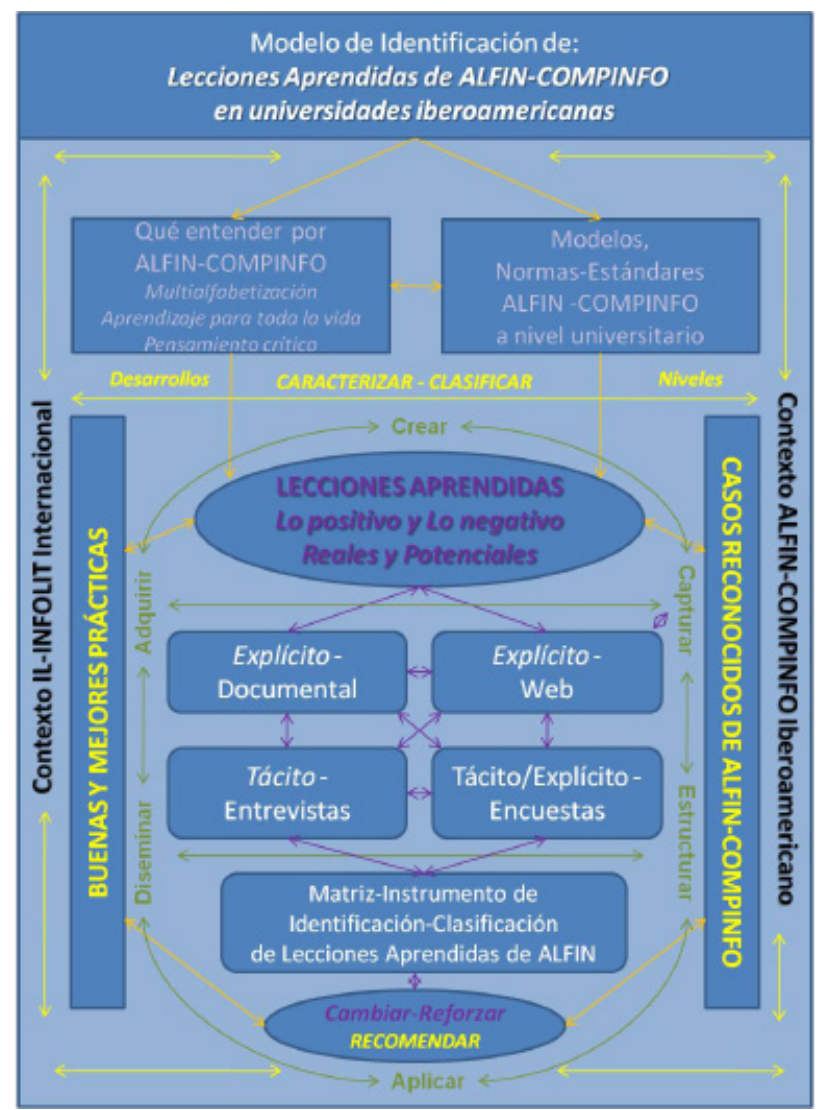




\section{RESULTADOS}

Es imposible presentar con amplitud los resultados particulares de cada uno de los métodos empleados, pero a modo de síntesis ofrecemos estos resultados, primero de manera gráfica general para todas las estrategias seguidas (tabla I), y luego de manera descriptiva y específica para cada una de ellas.
Análisis de contenido de la literatura publicada sobre programas de ALFIN en Iberoamérica

Se analizaron 1278 documentos, de un total de 1351 referenciados desde el Wiki-Repositorio ALFIN / Iberoamérica ${ }^{4}$ (figura 2).

Tabla I. Síntesis casos identificados y casos capturados

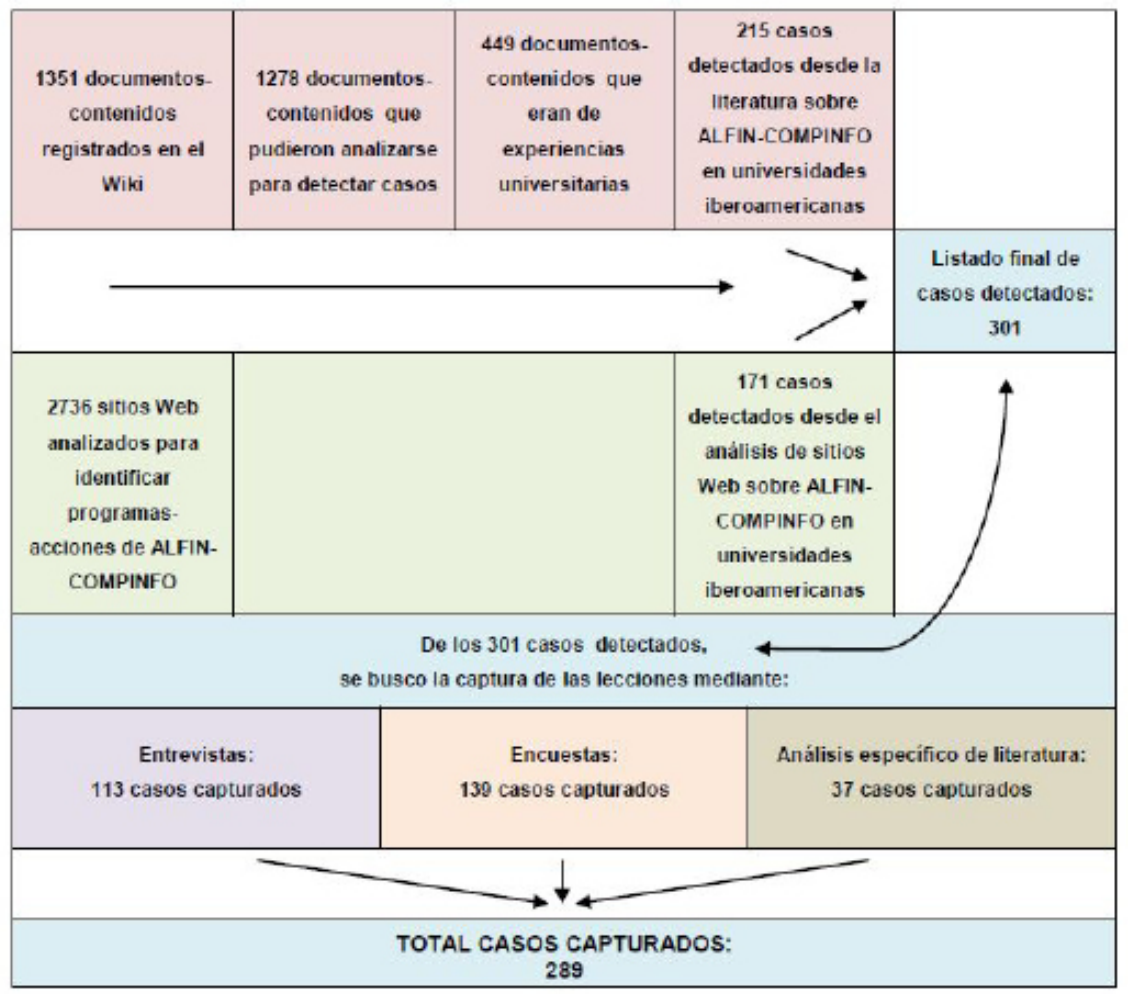

Figura 2. Distribución porcentual de los contenidos analizados (1278) según su orientación a lo teórico o lo teórico-práctico y la tipología de biblioteca-lugar de aplicación de ALFIN

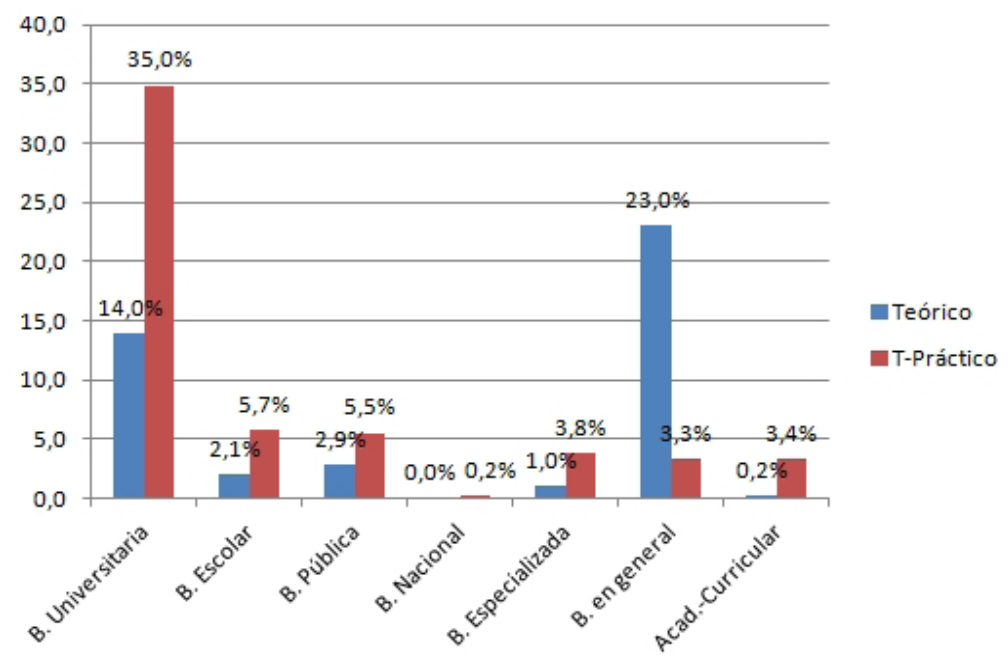


Como puede observarse, el 35\% de los documentos analizados son de orientación teórico-práctica y aplicados a bibliotecas universitarias (457 documentos), y un $3.4 \%$ están centrados en el ámbito académico universitario (42 documentos). Si se analizan estadísticamente estos 499 documentos, que son los de interés directo para esta investigación, se evidencia la tipología documental que se presenta en la figura 3.

Como consecuencia de este análisis de contenido documental, se identificaron 215 casos de programas de ALFIN en universidades iberoamericanas (figura 4) ${ }^{5}$.

Figura 3. Cantidad y tipo de contenidos (499) de orientación teórico-práctica y tipología-lugar de aplicación de ALFIN a nivel de bibliotecas universitarias y programas académicos-curriculares universitarios

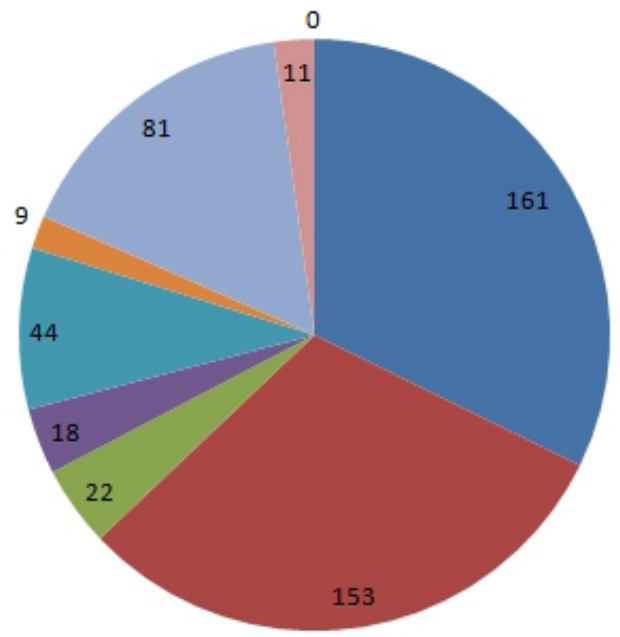

\author{
Artículos \\ Ponencias \\ - Libros/Capítulos \\ - Trabajos de grado-Pregrado \\ - Tesis-Posgrado \\ Declaraciones, modelos... \\ aresentaciones \\ Videos \\ Audios
}

Figura 4. Cantidad de contenidos (499) de orientación teórico-práctica y país de procedencia

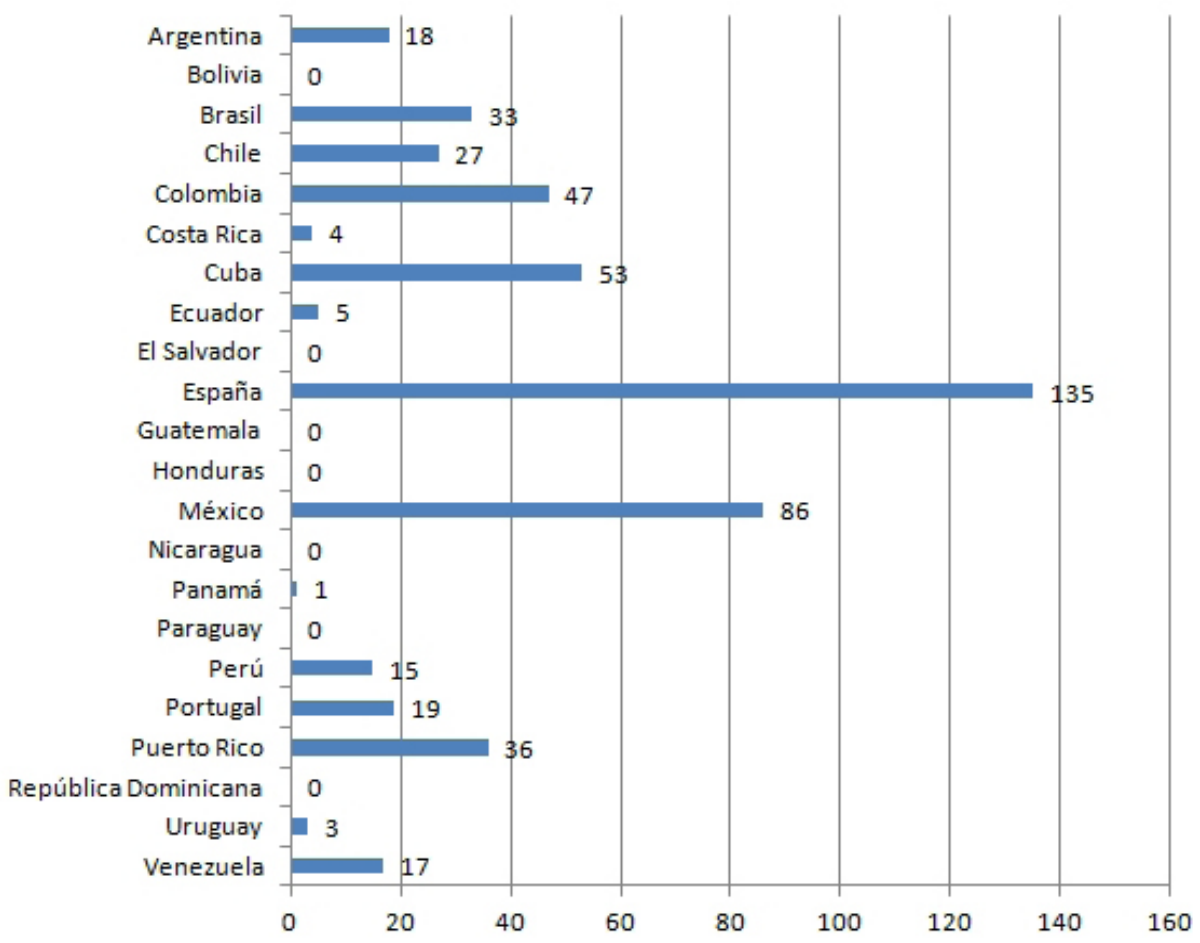


Análisis de contenido de los sitios Web de las bibliotecas universitarias iberoamericanas

La metodología descrita anteriormente, nos permitió desde el listado inicial generar una base de datos ${ }^{6}$ para cada país tanto con sus universidades públicas o privadas, como con sus otras IES (Institutos Tecnológicos, Institutos Técnicos y otros, según la denominación y estructura de la Educación Superior en cada país), que incluía sus bibliotecas universitarias y sus sitios Web (tabla II).

Esta base de datos, con un total de 2736 bibliotecas de universidades-IES iberoamericanas, recoge en una ficha normalizada los datos básicos de cada biblioteca, como url e información sobre ALFIN, además de otras variables, especialmente la de estructuración del programa y el nivel de desarrollo de ALFIN (figura 5).

Tabla II. Sitios Web analizados de bibliotecas de Universidades-IES iberoamericanas según tipología de pública o privada-particular

\begin{tabular}{|c|c|c|c|c|}
\hline Países & $\begin{array}{c}\text { No. de } \\
\text { Universidades-IES }\end{array}$ & Públicas & Privadas/Particulares & $\begin{array}{l}\text { Porcentaje por país } \\
\text { del total analizado }\end{array}$ \\
\hline Argentina & 131 & 66 & 65 & $4,8 \%$ \\
\hline Bolivia & 69 & 16 & 53 & $2,5 \%$ \\
\hline Brasil & 465 & 224 & 241 & $17,0 \%$ \\
\hline Chile & 79 & 21 & 58 & $2,9 \%$ \\
\hline Colombia & 337 & 106 & 231 & $12,3 \%$ \\
\hline Costa Rica & 75 & 19 & 56 & $2,7 \%$ \\
\hline Cuba & 36 & 36 & 0 & $1,3 \%$ \\
\hline Ecuador & 82 & 30 & 52 & $3,0 \%$ \\
\hline El Salvador & 49 & 4 & 45 & $1,8 \%$ \\
\hline España & 131 & 50 & 81 & $4,8 \%$ \\
\hline Guatemala & 54 & 10 & 44 & $2,0 \%$ \\
\hline Honduras & 38 & 12 & 26 & $1,4 \%$ \\
\hline México & 532 & 260 & 272 & $19,4 \%$ \\
\hline Nicaragua & 103 & 33 & 70 & $3,8 \%$ \\
\hline Panamá & 57 & 20 & 37 & $2,1 \%$ \\
\hline Paraguay & 59 & 6 & 53 & $2,2 \%$ \\
\hline Perú & 106 & 36 & 70 & $3,9 \%$ \\
\hline Portugal & 82 & 39 & 43 & $3,0 \%$ \\
\hline Puerto Rico & 48 & 10 & 38 & $1,8 \%$ \\
\hline Rep. Dominicana & 48 & 2 & 46 & $1,8 \%$ \\
\hline Uruguay & 41 & 14 & 27 & $1,5 \%$ \\
\hline Venezuela & 114 & 67 & 47 & $4,2 \%$ \\
\hline TOTALES & 2736 & 1081 & 1655 & \\
\hline
\end{tabular}

Figura 5. Niveles de incorporación de ALFIN en universidades ${ }^{7}$

\begin{tabular}{|c|c|c|}
\hline 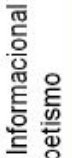 & 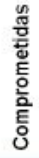 & $\begin{array}{l}\text { Alfabetización Informacional. Nivel 2: cursos desde la biblioteca para formar en } \\
\text { competencias informacionales: Io instrumental + aprendizaje para toda la vida + } \\
\text { pensamiento critico; y cursos/módulos especificos inmersos oficialmente en los } \\
\text { curriculos de distintos programas academicos-carreras para formar de manera } \\
\text { transversal y disciplinaren esas competencias }\end{array}$ \\
\hline 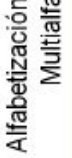 & 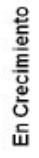 & $\begin{array}{l}\text { Alfabetización Informacional. Nivel 1: cursos desde la biblioteca para formar en } \\
\text { competencias informacionales: lo instrumental }+ \text { aprendizaje para toda la vida + } \\
\text { pensamiento critico }\end{array}$ \\
\hline 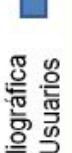 & $\begin{array}{l}\text { 윰 } \\
\frac{\text { 을 }}{\text { 으 }}\end{array}$ & $\begin{array}{l}\text { Formación de Usuarios. Nivel } 1 \text { : capacitación en servicios generales de la } \\
\text { biblioteca y algunos cursos -muy instrumentales- para búsqueda de información: } \\
\text { utilización de catálogos/bases de datos, aunque se comienza a analizar la necesidad } \\
\text { de cambio de esta formación tradicional y a trabajar las demás competencias }\end{array}$ \\
\hline 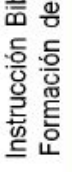 & 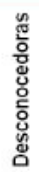 & Formación de Usuarios. Nivel 2: solo capacitación para el uso del catálogo \\
\hline
\end{tabular}


Tras el análisis de los sitios de bibliotecas universitarias e integrando los resultados de los 22 países iberoamericanos estudiados, se presenta esta realidad $^{8}$ respecto a los niveles de incorporación de ALFIN $^{9}$ (tabla III)

En suma, se han identificado 171 casos con niveles de desarrollo ALFIN AI1 -en crecimiento- y AI2 -comprometidas- que representan mayores posibilidades de sistematización y reflexión de su experiencia formadora en ALFIN.

Si integramos la información disponible en el análisis de la literatura y de los sitios Web, y depuramos los casos que se duplicaban en ambas fases (85 casos), nos encontramos con un listado final de 301 casos, a los que se aplicó la metodología cualitativa centrada en entrevistas y encuestas para capturar y categorizar dichas lecciones aprendidas.

Entrevistas a coordinadores $\mathrm{y} / \mathrm{o}$ formadores de ALFIN Iberoamérica

De una manera más cercana, se pretende conocer las experiencias de los casos detectados desde las dos fases anteriores, y a partir de los postulados del análisis de contenido cualitativo y de la teoría fundamentada se recogen esas LA conforme a la matriz ya validada (figura 1, parte inferior).

En este sentido, la asistencia a talleres, cursos, seminarios y las visitas programadas a bibliotecas de distintos países iberoamericanos, permitió realizar 113 entrevistas a coordinadores y/o formadores de los programas de ALFIN de universidades iberoamericanas, distribuidas por casos de país, así: Argentina: 4, Brasil: 4, Chile: 5, Colombia: 14, Costa Rica: 3, Cuba: 8, España: 35, México: 19, Perú: 3, Portugal: 5, Puerto Rico: 7, Uruguay: 1, Venezuela: 5.

\section{Encuestas a coordinadores $\mathrm{y} / \mathrm{o}$ formadores} de ALFIN Iberoamérica

Para finalizar la recogida de datos y poder triangular toda la información, se empleó una encuesta online a aquellas universidades identificadas que no pudieron participar en las entrevistas, con el propósito de poder capturar de manera indirecta sus LA. Se logró capturar 139 casos de programas

Tabla III. Total de bibliotecas universitarias analizadas y niveles de incorporación por país

\begin{tabular}{|c|c|c|c|c|c|}
\hline País & No presenta & FU2 & FU1 & AI1 & AI2 \\
\hline Argentina & 61 & 40 & 20 & 9 & 1 \\
\hline Bolivia & 56 & 12 & 1 & 0 & 0 \\
\hline Brasil & 216 & 121 & 95 & 30 & 3 \\
\hline Chile & 34 & 23 & 9 & 11 & 2 \\
\hline Colombia & 262 & 37 & 22 & 11 & 5 \\
\hline Costa Rica & 48 & 13 & 9 & 3 & 2 \\
\hline Cuba & 8 & 4 & 12 & 8 & 4 \\
\hline Ecuador & 63 & 10 & 9 & 0 & 0 \\
\hline El Salvador & 27 & 16 & 5 & 1 & 0 \\
\hline España & 55 & 18 & 29 & 19 & 10 \\
\hline Guatemala & 37 & 15 & 2 & 0 & 0 \\
\hline Honduras & 25 & 9 & 1 & 3 & 0 \\
\hline México & 460 & 34 & 18 & 15 & 5 \\
\hline Nicaragua & 87 & 3 & 13 & 0 & 0 \\
\hline Panamá & 44 & 5 & 8 & 0 & 0 \\
\hline Paraguay & 50 & 6 & 3 & 0 & 0 \\
\hline Perú & 80 & 13 & 8 & 5 & 0 \\
\hline Portugal & 45 & 11 & 19 & 5 & 2 \\
\hline Puerto Rico & 24 & 4 & 8 & 9 & 3 \\
\hline República Dominicana & 31 & 9 & 8 & 0 & 0 \\
\hline Uruguay & 28 & 3 & 10 & 0 & 0 \\
\hline Venezuela & 92 & 15 & 2 & 5 & 0 \\
\hline TOTALES & 1833 & 421 & 311 & 134 & 37 \\
\hline
\end{tabular}


universitarios de ALFIN, distribuidas por casos de país, así: Argentina: 8, Brasil: 19, Chile: 9, Colombia: 12, Costa Rica: 2, Cuba: 10, España: 29, Honduras: 1, México: 20, Perú: 5, Portugal: 5, Puerto Rico: 9, Uruguay: 1, Venezuela: 5.

\section{Análisis de contenido de la literatura específica de casos de ALFIN}

Mediante el análisis específico de los casos registrados inicialmente desde la literatura, se identificaron las lecciones de 37 de los 49 casos, que de otra forma no habrían podido ser capturados ya que ni por las entrevistas ni en la encuesta se pudo obtener su participación.

\section{75 LECCIONES APRENDIDAS}

En síntesis, el número total de casos sobre los que efectivamente se hizo la captura de las LA en consonancia con la triangulación de métodos empleados fue de 289 (113 casos por entrevista + 139 por encuesta + 37 por análisis específico de literatura). Tras un exhaustivo análisis cualitativo de los contenidos registrados, se sintetizaron en 75 lecciones aprendidas, organizadas en cuatro categorías que retoman los tres ámbitos que propone Gratch-Lindauer (2004), además de incorporar la evaluación como proceso continuo de un ciclo.

Estas LA se presentan numeradas para facilitar su lectura, pero no implican prioridad, y su redacción en infinitivo, debe entenderse como acciones que desde los casos se recomiendan para lograr mejores resultados en los programas de ALFIN en el contexto social y organizacional, y en los procesos de enseñanza-investigación, aprendizaje y evaluación de la calidad y mejora continua.

A su vez, para algunas de estas lecciones, no en todas, ya que la extensión de este artículo no lo permite, se incorporan algunos ejemplos y se referencian algunos de los casos que reflejan la aplicación de las mismas en el contexto de las bibliotecas-universidades iberoamericanas, cuyas notas pueden ubicarse al final de este artículo.

Es evidente que muchos otros casos de bibliotecas-universidades que han vivido y son ejemplo de tal o cual LA no están referenciados, pero ello no quiere decir que no sean también una evidencia de dicha lección.

Lecciones aprendidas relativas al contexto social y organizacional específico

LA 1) Vincular el programa de ALFIN con la misión y visión institucional y/o con las políticas de información y educativas nacionales.

LA 2) Trabajar en red como instituciones educativas, como bibliotecas universitarias líderes, para que en las políticas educativas, informativas $\mathrm{y} / \mathrm{o}$ tecnológicas locales, regionales o nacionales donde aún no está presente la ALFIN, sea considerada como una competencia fundamental a incorporarse de múltiples maneras según cada contexto ${ }^{10}$.
LA 3) Ser considerada la formación en competencias informacionales en los Planes de Desarrollo, Estratégicos, Operacionales, de Acción, de Docencia-Investigación de la institución ${ }^{11}$.

LA 4) Resaltar-Destacar por parte de las directivas universitarias, de Facultad o de Dependencia administrativa, la importancia de este programa y las competencias que propone fomentar y ayudar a formar en información.

LA 5) Soportar la planificación del programa considerando una definición concreta de ALFIN y los Modelos y Normas-Estándares existentes.

LA 6) Sustentar y resaltar la importancia de la formación en competencias informacionales ante directivas, profesores-investigadores y estudiantes a partir de documentos internacionales que desde el ámbito educativo e informacional avalan estas competencias, como parte de la educación actual y futura que deben ofrecer las universidades y poseer todo profesional, ante los requerimientos de la sociedad de la información ${ }^{12}$

LA 7) Conectar la ALFIN como parte fundamental de un currículo universitario que se representa en diferentes modalidades y mediaciones de enseñanza-aprendizaje.

LA 8) Adaptar el programa a las características estructurales, funcionales y curriculares institucionales.

LA 9) Comprometer en el buen desarrollo del programa a todos los grupos integrantes de una comunidad universitaria.

LA 10) Lograr que en las universidades donde haya Facultad de Bibliotecología-Documentación se realice un trabajo de apoyo conjunto en docencia, investigación y/o extensión, entre ésta y el Sistema de Bibliotecas, o con otras Facultades relacionadas con esta temática (Educación, Informática, Comunicación, Idiomas, etc.), para que así el programa mejore y crezca gracias al trabajo interdisciplinario o surjan nuevas propuestas aplicadas que potencien la formación en estas competencias ${ }^{13}$.

LA 11) Anticipar los cambios, oportunidades y amenazas que tiene el programa.

LA 12) Contar con los recursos financieros, tecnológicos, locativos y documentales (fuentes de información) necesarios para el buen desarrollo del programa y aprovechar los recursos ya disponibles en la institución y con los que puede contar como apoyo (área de informática, de diseño gráfico, de comunicación, de pedagogía, etc.), y en los casos en que esto inicialmente no es posible, hacer gestión y mostrar resultados para poder conseguirlos, o en su defecto, ante las dificultades presupuestales o de otra índole que hagan que difícilmente se tenga acceso a esos recursos, ser creativos y flexibles buscando siempre alcanzar los objetivos de la mejor manera. 
LA 13) Generar programas integrados (multialfabetismo) o nuevos espacios de acceso a la información y la formación (Bibliotecas híbridas, CRAI, CREA, etc.) entre distintas dependencias universitarias que potencien la formación necesaria ante los requerimientos de la sociedad actual ${ }^{14}$.

LA 14) Divulgar institucionalmente de manera constante los beneficios y logros de este programa.

LA 15) Acompañar el desarrollo del programa de ALFIN de un proceso estratégico y continuo de divulgación y mercadeo del mismo, utilizando diferentes medios y estrategias (desde avisos publicitarios y comunicativos masivos hasta el contacto uno a uno con estudiantes y profesores), buscando que los distintos públicos-agentes que tienen interrelación con la biblioteca o con el área de la Facultad a cargo de esta formación conozcan cada día más esta oferta formativa y su importancia ${ }^{15}$.

LA 16) Vincular a los profesores conscientes de la importancia de la gestión de información de calidad, al desarrollo del programa de ALFIN (especialmente detectando a los que han tenido la posibilidad de estudiar su maestría o doctorado en países con desarrollos significativos en IL-INFOLIT), para que actúen como facilitadores o divulgadores del mismo, al ser los principales aliados para lograr un mayor posicionamiento y evitar las actitudes reacias o desconocedoras de su importancia de otros profesores o de los estudiantes.

LA 17) Identificar, cuando hay universidades de gran tamaño y con múltiples bibliotecas en las que no es posible realizar por cuestión de alcance, recursos o de contexto un programa general de ALFIN para toda la universidad o sistema de bibliotecas, cuáles bibliotecas tienen adelantos significativos en su accionar de formación en ALFIN y que las mismas lideren o sean las principales orientadoras de otras bibliotecas y bibliotecarios interesados en ofrecer esta formación en su misma institución ${ }^{16}$.

LA 18) Generar un clima organizacional propicio en las bibliotecas o Facultades, para que los coordinadores y/o facilitadores de ALFIN, en relación con otros bibliotecarios, las directivas, los profesoresinvestigadores, puedan desarrollar toda su labor de formación en competencias informacionales, evitando los recelos y las posiciones reacias a la necesidad de estos nuevos aprendizajes.

LA 19) Posicionar más institucionalmente a la biblioteca, y a los profesionales de la información al interior de la Universidad por su contribución académico-científica, considerando sus aportes significativos a la formación en las competencias necesarias para la educación del siglo XXI, gracias a los programas de ALFIN

LA 20) Actualizar los currículos de formación de los bibliotecólogos, documentalistas y profesionales de la información para que ALFIN sea una temática de formación tanto teórico-conceptual como práctica en relación con elementos tecno- lógicos y pedagógico-didácticos, y de esa manera sea una verdadera competencia de los futuros profesionales de esta área del conocimiento, y una competencia a fortalecer, mediante la educación continua-posgrados, de los ya graduados ${ }^{17}$.

\section{Lecciones aprendidas relativas a los procesos de enseñanza e investigación}

LA 21) Tener siempre presente la historia y resultados de los procesos formativos vividos previamente (instrucción bibliográfica, formación de usuarios tradicional) y compararlos con lo que implica hoy formar en ALFIN, para de esa manera aprender lo positivo de esas experiencias y no cometer errores anteriores, y a su vez, marcar y evidenciar tanto en lo teórico-conceptual como en lo práctico, lo que diferencia a esta actual formación de la que se realizaba meses 0 años atrás ${ }^{18}$.

LA 22) Desarrollar el programa considerando las características particulares y contextuales (sociodemográficas) de los grupos-individuos que participan (estudiantes, profesores, investigadores, empleados, egresados) y los niveles educativos: pregrado, posgrado, educación continua; para ajustarlo a diferentes niveles, tiempos, intensidad, metodología y número de participantes (cobertura).

LA 23) Considerar todas las etapas, niveles, macro-competencias que implica un programa integral de ALFIN tanto en lo práctico como en lo teórico: necesidad de información, localización, valoración, organización, uso, comunicación y ética, evaluación y autoevaluación, etc.; y tener muy presente que el trabajo de todas estas competencias puede hacerse en diferentes etapas-períodos de acuerdo a las necesidades, intereses o la base de conocimientos que en un momento dado puedan tener los participantes y así ir facilitando un aprendizaje más gradual ${ }^{19}$.

LA 24) Desarrollar un plan administrativo-operativo y pedagógico-didáctico basado en competencias, para lograr los resultados esperados por el programa ${ }^{20}$

LA 25) Definir criterios, indicadores e instrumentos para la evaluación continua del programa.

LA 26) Trabajar conjuntamente docentes, investigadores, bibliotecólogos y coordinadores académicos, en la planeación, ejecución y evaluación del Programa.

LA 27) Utilizar diferentes medios (multimodalidad), espacios (físicos y/o virtuales: plataforma elearning) y metodologías de enseñanza centradas en el estudiante (grupal-personalizada; real-simulada), para la formación en estas competencias ${ }^{21}$.

LA 28) Identificar las herramientas Web 2.0 como medio de aprendizaje de competencias informacionales y como fuente de localización, organización y evaluación de información ${ }^{22}$. 
LA 29) Integrar las competencias esperadas en la formación curricular universitaria y las disciplinares, con las que implica la ALFIN, mediante: cursos, módulos y/o actividades concretas y flexibles; obligatorias u optativas; transversales o disciplinares $^{23}$.

LA 30) Realizar estudios sobre el currículum y el plan de estudios de los diferentes programas académicos para detectar puertas de entrada para ofrecer a esos programas, asignaturas y/o profesores que directa o indirectamente dan una gran importancia a la gestión de información, una oferta formativa según distintas posibilidades: charlas, talleres, tutoriales, módulos, cursos completos, etc.

LA 31) Aprovechar todas las oportunidades que las distintas Facultades puedan dar para la presencia, crecimiento y/o integración curricular o no del programa de ALFIN, aunque ello implique acciones distintas dentro del programa y que el mismo deba ser siempre flexible e innovador, pero teniendo claro que el objetivo general es la adquisición de las competencias informacionales, y los específicos, es la adquisición de cada una de las subcompetencias.

LA 32) Buscar alternativas para que la formación en ALFIN Ilegue a la mayor cantidad de estudiantes directa o indirectamente cuando son universidades de gran tamaño, bien sea mediante la estrategia de formar preferencialmente a los profesores y que éstos lo repliquen a los estudiantes, o gracias a la utilización de diferentes opciones de formación mediadas por tecnologías: cursos virtuales, tutoriales, manuales y guías interactivas, juegos y concursos, webquest, tours, videos, podcast, tips vía celular, apps, redes sociales, etc. ${ }^{24}$

LA 33) Adecuar la formación en competencias informacionales a los intereses de otros grupos de la comunidad universitaria, para así tener más aliados e impacto en la formación de estas competencias, específicamente con los profesores-investigadores, por medio de temáticas relativas a mejorar procesos de investigación, de visibilidad de sus publicaciones, de derechos de autor, o en el caso del personal administrativo, en aspectos relativos a hacer más eficiente su labor ${ }^{25}$.

LA 34) Pensar la oferta de formación en ALFIN considerando también a los grupos poblacionales que tiene una universidad y que presentan algún tipo de discapacidad o una característica multicultural significativa, para así ajustar esa formación a las realidades de esa población, y con ello garantizar tanto el acceso a la información como a la formación, para toda la comunidad universitaria.

LA 35) Considerar que si se tiene una biblioteca virtual, hay muchos usuarios que solo tienen una relación de acceso virtual a la información, por lo que se hace necesario que el programa de ALFIN tenga un subprograma de formación exclusivamente virtual y adaptado a las características del medio, del tipo de usuarios, de la disponibilidad de tiempo y de las posibilidades didácticas de la no presencialidad $^{26}$.

LA 36) Tener conciencia que la formación en competencias informacionales no se da solo desde las acciones formales de formación, sino también desde la comunicación diaria con los usuarios por diferentes vías: servicio de referencia, préstamo, carteleras, avisos electrónicos, mensajes digitales y demás, por lo cual la ALFIN implica a toda la biblioteca y su personal: crear la biblioteca como un escenario alfabetizador informacionalmente.

LA 37) Relacionar y valorar previa y complementariamente los niveles de las otras competencias relacionadas con la ALFIN (alfabetización funcional, digital, en medios; conocimiento de otros idiomas) para identificar las potencialidades reales de los participantes de adquirir competencias informativas y diferenciarlas-relacionarlas de las competencias que implican esas otras alfabetizaciones (multialfabetismo).

LA 38) Trabajar el diseño instruccional y los objetos de información y aprendizaje de ALFIN, desde que el contexto lo posibilite, considerando la perspectiva del acceso abierto y de los repositorios, con el objetivo que esta formación, esos recursos informativo-formativos, puedan utilizarse en cualquier momento y beneficiar a más universitarios, a más ciudadanos, y cumplir así una función social de información-formación, además de posibilitar un intercambio mayor de experiencias con otras bibliotecas y la validación de los mismos en distintos contextos.

LA 39) Conseguir la continuidad y el desarrollo gradual del programa para una mayor cobertura en la formación y un impacto significativo, al abarcar mayor cantidad de usuarios: estudiantes, profesores, investigadores, empleados y/o egresados.

LA 40) Lograr la actualización constante de los contenidos, medios didácticos-tecnológicos y de los ejemplos disciplinares en relación con la información, para generar una mejor respuesta de los participantes y una mayor motivación para el aprendizaje.

LA 41) Considerar como temática de formación, la gestión de información, tanto de fuentes físicas o digitales, teniendo en cuenta sus potencialidades y las realidades contextuales que facilitan o no el acceso y uso de determinadas fuentes.

LA 42) Tener claro que la formación en competencias informacionales y el desarrollo de estas competencias por parte de los estudiantes dentro de los años de desarrollo de su formación universitaria, dependerá tanto de la organización de cursos/actividades concretas para esta formación, como de la conciencia que se tenga de la importancia de estas competencias por parte de los profesores y sus propios niveles de competencia en la gestión de información académica-científica. 
LA 43) Desarrollar los programas de ALFIN no solo desde la perspectiva de educación formal en la universidad y desde el accionar de las bibliotecas académicas, sino también desde los programas de actualización, de educación continua, de extensión que requieren las organizaciones (empresas o instituciones sociales y comunidades) para apoyar la formación en las competencias laborales y profesionales que exige la sociedad actual, en relación con la información y la gestión del conocimiento y el capital social, y de esta forma cumplir con la responsabilidad social de toda universidad o biblioteca, a su vez, en los casos que sea pertinente, tener una fuente de financiación alternativa para la misma sostenibilidad económica del programa.

LA 44) Tomar conciencia de la necesidad de tener una coordinación específica del programa y que el formador debe tener un perfil que implica el dominio de competencias informacionales-informáticas como pedagógicas-didácticas, para ser un buen facilitador de aprendizajes.

\section{Lecciones aprendidas relativas a los procesos} de aprendizaje

LA 45) Identificar las competencias informacionales como un aspecto fundamental tanto para un mejor rendimiento académico, científico y laboral como para el aprendizaje permanente y colaborativo, para el pensamiento crítico...

LA 46) Valorar los conocimientos y experiencias previas de los participantes y sus intereses, como fuente de aprendizaje significativo ${ }^{27}$

LA 47) Reconocer las diferencias y exigencias disciplinares de los participantes en relación con la información y las distintas expectativas de formación según las culturas académicas.

LA 48) Diagnosticar y reconocer los ritmos y estilos de aprendizaje de los participantes, sus ritmos y estilos de comportamiento Informacional (novato, intermedio, avanzado u otros) y propender por su aprendizaje autónomo, siendo conscientes que los cambios generacionales, y por ende, de cultura informacional son cada vez más cortos, por tanto, esos diagnósticos, esos estudios del comportamiento informacional, deben ser continuos y periódicos para que la formación se ajuste a esas realidades cambiantes.

LA 49) Mejorar la motivación de los estudiantes para la formación en competencias informacionales, no solo a partir de temas de interés académico sino también de los temas de interés personal y social que generen más motivación inicial hacia el aprendizaje de estas competencias, y así, resaltar su importancia, no solo exclusivamente para su vida profesional, científica y académica, sino también para sus otros roles como persona-ciudadano.

LA 50) Presentar claramente a los estudiantes u otros grupos que vayan a recibir la formación en ALFIN los objetivos y alcances de esta forma- ción, para evitar mayores o menores expectativas y compromisos, y a su vez, evidenciar que esta formación se puede asumir de manera gradual por diferentes niveles o subcompetencias (si está estructurado de esa manera el programa), y que cada una es muy importante.

LA 51) Vincular la formación de ALFIN que estén desarrollando los participantes, con trabajos concretos de sus cursos, trabajos de investigación, desempeño docente-científico y/o administrativo.

LA 52) Desarrollar las actividades formativas del programa con didácticas activas específicas (aprendizaje basado en proyectos, en problemas, en casos, concursos, etc.) que faciliten, motiven y generen retos en los estudiantes para el aprendizaje de las competencias informacionales ${ }^{28}$.

LA 53) Presentar y motivar la adquisición de las competencias informacionales como una labor de disfrute, de reto, de espíritu científico, para ubicar la información precisa y utilizarla para beneficio personal, académico, profesional y/o social.

LA 54) Tender a que el lenguaje oral, textual y/o audiovisual que implica la formación en ALFIN por diferentes modalidades, medios o contenidos, sea acorde a los conocimientos, nivel generacional y/o cultura de los destinatarios, para de esa manera garantizar que la formación sea más comprensible y apropiada, sin caer en excesivos informalismos o formalismos que generen lejanía o rechazo con las temáticas y competencias sobre las que se están formando, y hasta por el nombre mismo del programa.

LA 55) Identificar que los cursos y actividades formativas en competencias informacionales permiten mejores resultados de aprendizaje cuando se desarrollan desde una perspectiva más segmentada y personalizada, que implique grupos no muy grandes y el trabajo en intereses particulares y disciplinares, aunque con una amplia cobertura que abarque a la mayor cantidad de integrantes de la comunidad universitaria que requiere esta formación.

LA 56) Definir criterios, indicadores e instrumentos para la evaluación formativa y sumativa, opcional u obligatoria, y de impacto, de los participantes del programa.

LA 57) Permitir y valorar la autoevaluación de los participantes (de entrada, durante la formación y al final) como parte del proceso formativo, aunque esta valoración, retroalimentación, no puede ser la única medición para detectar si se adquirieron o no estas competencias, si el proceso formativo fue o no exitoso; por tanto, se hace necesario tener otras metodologías e instrumentos de medición, más de los aprendizajes que de los niveles de satisfacción.

LA 58) Garantizar que los aprendices puedan tener, tras pasar por las diferentes ofertas formati- 
vas, un espacio presencial o virtual donde puedan actualizarse, recibir respuesta a alguna duda, o recordar y autoformarse en alguna de las subcompetencias informacionales, bien sea en un aspecto teórico-conceptual o en uno aplicado-instrumental, ya que es clave que haya espacios para que la formación en ALFIN sea continua al interior de la universidad.

LA 59) Potenciar la creación de comunidades de aprendizaje o de redes sociales, presenciales o virtuales, que posibiliten el intercambio de experiencias entre los que han recibido la formación en estas competencias, ya que puede potenciar entre los mismos formandos, con la mediación de los bibliotecarios y/o profesores, que estos sean formadores de otros colegas y pares considerando que la cercanía temática, generacional y demás, ayuda a desarrollar otras alternativas de aprendizaje y a la actualización continua en cada una de las subcompetencias que implica la ALFIN.

LA 60) Facilitar espacios divulgativos, uno a uno o uno a muchos, a quienes se han beneficiado de esta formación, para que sean los principales divulgadores del Programa, ya que la mejor publicidad es la que viene de un referente semejante.

LA 61) Vincular los procesos de formación en ALFIN a nivel universitario (pregrado y posgrado) con los procesos de formación de estas competencias en otros niveles educativos previos (primaria y secundaria) y cuando estos no están presentes; tener un accionar claro de extensión y aporte social para facilitar la adquisición de las competencias informacionales, según los niveles pertinentes, de esos otros ámbitos educativos previos ${ }^{29}$.

Lecciones aprendidas relativas a los procesos de evaluación de la calidad y mejora continua

LA 62) Capacitar permanentemente a los formadores (bibliotecólogos, informáticos, profesores de diferentes disciplinas, etc.) y lograr el acompañamiento de expertos en aspectos pedagógicos, tecnológicos e informacionales, buscando generar a su vez, una comunidad de aprendizaje en esta temática formativa.

LA 63) Facilitar la formación graduada o posgraduada de los bibliotecarios interesados o ya formadores en ALFIN, para que de una manera más formal y permanente logren otras competencias necesarias, además de las informativodocumentales, para ser mejores formadores (pedagogía-didáctica, tecnología, comunicación, idiomas, etc.), a la vez que al posibilitarse su participación en esos otros espacios universitarios, estos sean una oportunidad que: posibilite contactos con otros miembros de la comunidad universitaria, dar a conocer el programa, generar trabajos interdisciplinarios y lograr que haya más conciencia de los nuevos roles del bibliotecario, especialmente el rol educativo, que siempre ha estado pero que ante las exigencias de la sociedad de la información, es cada vez más necesario ${ }^{30}$.

LA 64) Consultar constantemente los avances en ALFIN como temática teórico-conceptual y aplicada a nivel global o local.

LA 65) Compartir e intercambiar de forma periódica (colaborativamente presencial o virtualmente) información, métodos y planes con otros colegas coordinadores-formadores en ALFIN de distintos contextos y entidades educativas, y propiciar la generación y participación activa en redes locales, regionales, nacionales o internacionales de formadores en ALFIN (sea desde asociaciones o redes ya existentes, o desde la creación de nuevas) para así apoyarse mutuamente, generar consorcios formativos, espacios de reconocimientos y premios a labores destacadas, y momentos formales y continuos de intercambio (eventos) o de desarrollo de líneas, políticas y/o recomendaciones para las propias universidades o los ámbitos educativos, informativos y tecnológicos de los diferentes países.

LA 66) Generar procesos formales y continuos de benchmarking en diferentes contextos, para identificar casos de éxito en ALFIN y así aportar también al mejoramiento permanente del programa, a partir de las adaptaciones contextuales-organizacionales necesarias.

LA 67) Divulgar constantemente los resultados y avances del programa en diferentes publicaciones científicas y divulgativas ${ }^{31}$.

LA 68) Generar procesos formales de acompañamiento (mentoring), para que los nuevos formadores aprendan de manera más sistemática y estratégica de los formadores experimentados en ALFIN, y generar mecanismos para que los conocimientos, las lecciones aprendidas, de los formadores se socialicen y generen conocimientos en la actualidad y a futuro, y así garantizar que esas experiencias sean aprendidas por otros y no se pierdan, es decir, hacer gestión del conocimiento en los mismos programas de ALFIN.

LA 69) Evaluar en forma periódica el programa y sus coordinadores-formadores: a corto, mediano y largo plazo (impacto), y los procesos-resultados, para así alcanzar su mejoramiento continuo.

LA 70) Lograr la retroalimentación permanente de los participantes y acoger las sugerencias pertinentes.

LA 71) Identificar la necesidad de generar y medir con indicadores tanto cuantitativos como cualitativos, para evaluar secuencialmente el proceso y lograr buenos resultados del programa tanto a corto plazo como a mediano y largo plazo.

LA 72) Considerar metodologías y herramientas de evaluación externas (ya validadas) para adaptarlas al propio contexto, o generar las propias metodologías y herramientas de evaluación (y validarlas), para lograr de una manera continua y 
comparativa (entre facultades-escuelas, universidades, etc.), evidenciar la real adquisición de las competencias informacionales y el impacto académico, científico y social que éstas han posibilitado en quienes han recibido dicha formación.

LA 73) Facilitar procesos de certificación de competencias informacionales que posibiliten en el ámbito universitario, el acceso a determinados niveles curriculares; y en el ámbito organizacional y empresarial, participar en determinados cargos considerando su importancia en la educación y la producción en la sociedad actual.

LA 74) Vincular los procesos de gestión y certificación de la calidad y de acreditación que viven las bibliotecas y las universidades, a los programas de formación de ALFIN para facilitar y mejorar sus procesos gracias a la identificación de los mismos, la generación de procedimientos y guías, y el registro y la evaluación permanente de esos procesos y resultados.

LA 75) Reconocer por parte de los responsables universitarios los logros del programa, y estimular de diferentes maneras el buen desempeño de sus coordinadores $\mathrm{y} / \mathrm{o}$ facilitadores.

\section{CONCLUSIONES Y RECOMENDACIONES}

Si nos centramos en estas 75 LA, podemos concluir que la guía generada no es un documento cerrado, sino más bien el resultado global de la visión de diferentes participantes de instituciones universitarias iberoamericanas. Es un resultado dinámico, cambiante, por lo que desde su publicación en el Wiki ALFIN / Iberoamérica: http://alfiniberoamerica.wikispaces.com/*Lecciones+Aprendidas se busca facilitar su consulta y/o actualización por parte de todos los alfineros iberoamericanos, es decir, que sea una guía de construcción continua y colectiva en el futuro cercano. Es probable que haya otras lecciones y casos valiosos sobre este tema que no ha sido posible tener en cuenta por circunstancias ajenas a nuestra voluntad.

De forma específica, si analizamos las cuatro categorías y sus correspondientes lecciones, podemos concluir que:

- Un programa de ALFIN debe estar planificado y estructurado tanto en lo estratégico-administrativo como en lo pedagógico-didáctico, y cuanto mejor sea dicho proceso los resultados pueden ser más positivos.

Sin embargo, considerando las respuestas y opiniones de los participantes que compartieron sus LA, se debe recomendar a los coordinadores $y / 0$ formadores que quieran iniciar un programa o mejorar la formación tradicional que hasta ahora han realizado, que sean proactivos pues el "exceso de análisis puede también producir parálisis". De manera, que aunque no esté totalmente listo todo y confirmado, se vayan realizando procesos de formación.
Dicha planificación es un proceso cambiante que debe basarse en las LA de la práctica de la enseñanza-aprendizaje de ALFIN, lo que requiere de una retroalimentación continua tanto de los formados (usuarios, estudiantes, etc.) como de los formadores (bibliotecarios, profesores, etc.), para obtener información valiosa sobre lo que se ha logrado y lo que se puede lograr.

- Para llevar a cabo un programa de ALFIN es importante contar con recursos propios y suficientes en cuanto a personal formador, tecnología, fuentes de información, espacios físicos, etc.

Desafortunadamente la realidad de muchos países, universidades y bibliotecas iberoamericanas no permiten en la actualidad, ni en un futuro cercano, tener todos los recursos que se necesitarían o quisieran, pero ello no puede ser un impedimento para realizar algunas acciones efectivas de formación.

Para suplir estas carencias en la medida de lo posible, se recomienda apostar por la creatividad (aprovechar al máximo los recursos que se tienen) y por la disposición a trabajar de manera colaborativa. Esto implica desde poder hacer un programa de formación con pocos recursos pero mucha didáctica, hasta hacerlo con recursos compartidos por otros (open access, creative commons), tratando que dichas acciones formativas se adapten al propio contexto (ejemplos, palabras específicas según el idioma y país de procedencia).

Incluso sería recomendable crear consorcios de formación, bien locales, nacionales y/o internacionales, para el crecimiento de estos programas en Iberoamérica.

- Las tecnologías cambian de manera constante, por lo que con seguridad serán necesarias nuevas opciones para mantener la visibilidad de lo que se hace en ALFIN.

Terminamos reafirmando la importancia crucial de que tanto los docentes como los profesionales y asociaciones asuman esta nueva perspectiva de trabajo, presenten nuevas formas de trabajo para que esta temática se actualice y siga siendo un tema de discusión, investigación y aplicación desde la bibliotecología-documentación o desde otras disciplinas. Además, sería recomendable reforzar el trabajo colaborativo entre los responsables de gestionar y mantener los recursos Web existentes sobre ALFIN.

\section{NOTAS}

[1] Para la captura de lecciones aprendidas, para su identificación y posterior transformación en buenas prácticas, además de las aportaciones generales de las perspectivas de la investigación mixta, diferentes autores han presentado distintos métodos e instrumentos aplicados y validados en distintos contextos, de los cuales esta investigación acoge algunos 
de ellos, con las debidas adaptaciones, ya que su utilización ha sido más desde el aspecto empresarial en la implementación de programas de Gestión de Conocimiento (Patton, 2001, 2002; Syed-Ikhsan y Rowland, 2004; Ahmad Sharif y otros, 2004; Wellman, 2007; Freeze y Kulkarni, 2007; Ontko y otros, 2007; Burley y Pandit, 2008; Roulston, K. y otros., 2008; Bartczak y otros, 2010; etc.)

[2] Habría que indicar en esta parte de la prueba piloto, como respuesta a por qué se eligió estos dos países y sus programas de ALFIN como muestra, que dicha respuesta tiene dos enfoques, uno teórico-conceptual y otro pragmático. Desde el teórico-conceptual, si comparamos la situación de los países iberoamericanos considerando la perspectiva que daba el repositorio INFOLIT Global de UNESCO, estos dos países estarían en un nivel medio de producción de contenidos (Uribe-Tirado, 2010), y por tanto, al ser una prueba piloto estos dos países estarían en un lugar intermedio. A su vez, la realidad de estos dos países, si tenemos en cuenta aspectos políticos, económicos, culturales, educativos, tecnológicos, tiene aspectos comunes (Idioma, Creatividad, Resiliencia, etc.), pero a su vez, muchas diferencias que permiten enmarcar el aspecto dinámico, y fenomenológico que tiene esta investigación. Por otro lado, desde el enfoque pragmático, un primer aspecto sería el tiempo y los recursos para su desarrollo, que implicaba para validar la versión inicial del Modelo, elegir solo un par de países. Como segundo aspecto, se valoró la posibilidad de estancia en Colombia y Cuba para realizar el trabajo de campo.

[3] Este proceso dio origen a diferentes recursos Web útiles para esta investigación, compartidos a través del blog ALFIN / Iberoamérica: http://alfiniberoamerica.blogspot.com/ y el Wiki ALFIN / Iberoamérica: http://alfiniberoamerica.wikispaces.com/

[4] A pesar de ser referenciados en el Wiki-Repositorio http://alfiniberoamerica.wikispaces.com/, hubo 73 contenidos que no pudieron analizarse, pues ni por fuentes de acceso abierto, ni por la consulta de bases de datos comerciales o de bibliografía disponible en el Sistema de Bibliotecas de la Universidad de Antioquia (Colombia) o de la Universidad de Granada (España), fue posible consultarlos a texto completo.

[5] Para conocer los listados que generó cada estrategia y otra información adicional, se puede consultar el texto completo de la investigación que dio origen a este artículo, en la recopilación de textos del autor en E-lis: http://bit. ly/12VWYgS o en la base de datos TESEO: https://www.educacion.gob.es/teseo/mostrarRef.do?ref = 1054962

[6] Esta base de datos se generó siguiendo la misma metodología para cada país. En primer lugar, se ubicaban los sitios Web oficiales relativos a universidades, a educación superior, en los cuales se tuviera referencia y/o acceso directo a los listados de Universidades-IES de dicho país y a sus sitios Web. En segundo lugar, para depurar y ampliar esos listados gubernamentales se recurrió a dos fuentes internacionales que posibilitan la identificación y el acceso a la dirección Web de cada universidad: Ranking Web de Universidades del Mundo del CCHS/CSIC (http://www.webometrics.info/es) y Portal estudiantil: El Altillo.com (http://www.altillo.com/universidades/index.asp)

[7] La numeración de los niveles es invertida en la parte de Formación de Usuarios y en la de Alfabetización Informacional, pensando en que la ALFIN va a seguir avanzando, va hacia el futuro, y llegará a un nivel 3, 4 ó 5, etc., a describir más adelante considerando las perspectivas de avance de esta temática (Multialfabetismo, Mooc, etc.), mientras que la formación de usuarios estaría en lo tradicional e implica lo pasado.
[8] Es importante indicar, al tener en cuenta este análisis de contenido Web, que esta categorización es una aproximación desde la información que tienen los sitios Web, pero en ningún momento supone categorizar a una u otra universidad en alguna de esas 4 categorías, ya que "no siempre en los sitios web se publica todo lo que se hace" o por el contrario "se publica más de lo que realmente se hace". Por tanto, este análisis es una fotografía en un momento dado que aporta una visión general del desarrollo que se estaría dando en la formación en ALFIN.

[9] El análisis detallado para los países con resultados específicos se ha publicado en diferentes revistas. Estos pueden consultarse en: http://alfiniberoamerica.blogspot. com/2012/06/analisis-de-alfin-para-las-bibliotecas.html

\section{Ejemplos de casos para algunas LA}

[10] Un ejemplo de esta lección es el trabajo que desde REBIUN (Red de Bibliotecas Universitarias de España) se viene haciendo para trabajar colaborativamente en la formación de competencias informacionales e informáticas en los grados: http://www.ci2.es/objetivos

[11] Un caso destacado de esta lección es la Universidad Nacional de Cuyo de Argentina donde la ALFIN es obligatoria y está reglamentada en documentos oficiales de su Rectorado: http://sid.uncu.edu.ar/sid/wp-content/uploads/2011/07/R_ RE_0718_20111.pdf

[12] En el caso de la Universidad Autónoma de Ciudad Juárez de México desde sus inicios ha tenido como referente las normas internacionales de ACRL-ALA que han sido adaptadas al propio contexto: http://bivir.uacj.mx/dhi/DocumentosBasicos/Default.htm

[13] En esta lección, la Universidad Carlos III de Madrid de España desde su Departamento de Documentación ha sido un ejemplo para otras Facultades/ Escuelas de esta área del conocimiento en Iberoamérica, por el trabajo directo de sus profesores en la formación ALFIN junto a los bibliotecarios: http://www.uc3m.es/portal/page/portal/biblioteca/ aprende usar/alfabetizacion_informacion. También destaca el proyecto PRODIC de la Escuela de Bibliotecología de la Universidad de la República de Uruguay, que ha apoyado la aplicación de ALFIN a nivel escolar considerando el Plan CEIBAL: http:// www.prodic.edu.uy/investigacion/alfabetizaci\%C3\%B3n-eninformaci\%C3\%B3n-y-competencias-lectoras; o el trabajo desde los grupos de investigación asociados a las respectivas Escuelas de Bibliotecología-Ciencia de la Información en Brasil de la Universidad Federal de Santa Catarina-Grupo GPCin: http://gpcin.webnode.com.br y de la Universidad Federal da Paraíba-Laboratório de Tecnologias Intelectuais (LTi): http:// http://dci.ccsa.ufpb.br/Iti/

[14] Para esta lección destacan algunas propuestas de CRAI especialmente a nivel de España, que han integrado entre sus tareas la formación en diferentes competencias, y la celebración de Jornadas específicas para dar a conocer sus trabajos: http://www.rebiun.org/documentos/Paginas/Jornadas-CRAI.aspx

[15] Muchas Universidades-Bibliotecas ven en la divulgación la clave para que la ALFIN sea aprovechada por su comunidad universitaria, entre los muchos casos existentes destaca la Universitat Rovira i Virgili de España: http://www. urv.cat/crai/que-us-oferim/formacio-competencies-nuclears/ index.html, y la Universidad EAFIT de Colombia: http://www. eafit.edu.co/biblioteca/servicios/formacion-usuarios/Paginas/ competencias-informacionales.aspx\#.Umf1vGcvq9s

[16] Para esta lección se pueden mencionar los casos de la UNAM de México y su biblioteca del Centro de Enseñanza de 
Lenguas Extranjeras: http://ced.cele.unam.mx/formaus_biblioteca/ o de la biblioteca de Ingeniería en la Universidad de Sao Paulo de Brasil: http://e-portfolios-sibi-usp.blogspot. com.es/

[17] El Caso de la Escuela Interamericana de Bibliotecología de la Universidad de Antioquia de Colombia sería un referente de esta lección donde tanto la Escuela como el Sistema de Bibliotecas han trabajado unidos los últimos años en la formación en ALFIN de los futuros profesionales: http://formacionbiblioteca.udea.edu.co/moodle/course/view.php?id=97 - https://sites.google.com/site/usuarioseib/ - http://formacionbiblioteca.udea.edu.co/moodle/ course/view.php?id=348

[18] Un ejemplo del reconocimiento de los procesos de formación como elemento clave para su actualización y mejora, es la línea de tiempo creada por la Universidad de Sevilla de España sobre sus programas de formación en ALFIN: http:// www.dipity.com/alfinbus/ALFIN/

[19] En cuanto a la parte específica de evaluación y autoevaluación de los aprendizajes, de las competencias adquiridas, podemos mencionar programas de ALFIN de bibliotecas concretas o programas para público universitario amplio y de alcance nacional o iberoamericano. Por ejemplo, en la guía de la Universidad Oberta de Cataluña de España cada módulo tiene actividades y test de autoevaluación: http://materials.cv.uoc. edu/continguts/ PID_00171989/index.html, y de manera semejante, el Centro Universitario de Ciencias Económico Administrativas de la Universidad de Guadalajara de México: http:// ceri.cucea.udg.mx/serviciosenlinea/prifesc/index.swf. También son destacables algunos instrumentos elaborados por profesores para autoevaluar-evaluar las competencias informacionales de los estudiantes universitarios, como IL-HUMASS: http:// www.mariapinto.es/alfin-humass/ e INFOLIT-TRANS: http:// www.infolitrans.edu.es/ infolitrans.php

[20] En lo referente a planes de ALFIN, varias bibliotecas universitarias iberoamericanas han sido muy conscientes de la importancia de documentar estos programas, como en el caso de España, la biblioteca de la Universidad de Jaén: http://www10.ujaen.es/conocenos/servicios-unidades/biblio/ alfabetizacion-informacional; de la Universidad de Las Palmas de Gran Canaria: http://biblioteca.ulpgc.es/formacion_rail; la Universidad Oberta de Catalunya: http://biblioteca.uoc.edu/ cat/index.html?serveis/serveis04; la Universidad de Almería: http://repositorio.ual.es/jspui/bitstream/10835/1587/1/IntegraCI2_UAL.pdf; y la Universidad de Cantabria: http://www. buc.unican.es/formacion. $Y$ en el caso de otros países iberoamericanos, la Universidad de Guadalajara-Centro Universitario de la Costa Sur de México: http://www.biblioteca.cucsur. udg.mx/desarrollo-de-habilidades-informativas; e INFOMEDUniversidad Virtual de Salud de Cuba: http://programafrida. net/ projects/projects/view/230 - http://aulauvs.sld.cu/course/category.php?id=71

[21] En lo correspondiente a formación apoyada en e-learning, uno de los casos más destacados es el de la biblioteca de la Universidad de La Laguna de España: http://www.bbtk.ull. es/view/institucional/bbtk/Competencias_informacionales/es, que ha sido referente para otros programas de ALFIN en Iberoamérica, y fundadora del Consorcio UNICI2; igualmente el de la UNED de España con cursos abiertos: http://ocw.innova. uned.es/competencias_BCA/curso/index.htm

[22] En cuanto a la utilización de herramientas de la Web 2.0 tanto como fuentes de información como medios de formación en ALFIN el trabajo de la biblioteca de la Universidad de Sevilla de España es uno de los más destacados y con mayor recorrido: http://bib.us.es/aprendizaje_investigacion/guias_tutoriales/index-ides-idweb.html. Igualmente se destacarían los casos de las bibliotecas de la Universidad de Zaragoza de España: http://blog.biblioteca.unizar.es/ cursos-formacion/, la Universidad de Valladolid de España: http://formacionbib.wordpress.com/post/ y la biblioteca de la Universidad de Chihuahua de México: http://fterrazas.wordpress.com/integracion-modelo-dhi/, además de la biblioteca de odontología de la Universidad Nacional de Cuyo de Argentina: http://blogs.unc.edu.ar/bcafo/formacion-de-usuarios/

[23] Un ejemplo de formación teniendo en cuenta la cultura disciplinar es el de El Colegio de México en el área de Ciencias Sociales y Humanidades: http://biblio.colmex.mx/ curso investigacion_documental/tutorial/default.htm, o el de la biblioteca de la Universidad de Lima en Perú: http://recursosdi. wikispaces.com/Recursos+de+informaci\%C3\%B3n+ acad\%C $3 \%$ A9mica+por+especialidad

[24] La utilización de estas distintas herramientas y recursos se ha ido dando gradualmente en la formación en ALFIN. En el caso específico de algunas de esas distintas herramientas y recursos, podemos destacar: las guías y tutoriales utilizados por la Universidad de Évora de Portugal: http:// www.bib.uevora.pt/guiaLI/, la Universidad de Barcelona de España: http://portal.uc3m.es/portal/page/portal/biblioteca/aprende_usar/autoformacion1, la Universidad Pompeu Fabra de España: http://www.upf.edu/bibtic/es/recursos/ treaca/ y el Instituto Tecnológico de Monterrey-ITESM de México: http://www.ruv.itesm.mx/cursos/ maestria/bib_digital/nivel1/; el podcast por la Universidad Metropolitana de Venezuela: http://agora.unimet.edu.ve/ens.php?e=77, la Universidad de Chile: http://informacionacademica.pbworks. com/w/page/19912880/Programa; el video por la Universidad Carlos III de Madrid: http://portal.uc3m.es/portal/page/ portal/biblioteca/ aprende_usar/autoformacion1; los juegosconcursos por la Universidad Autónoma de San Luis de Potosí de México con su Rally Virtual Académico: http://tzaloa.uaslp. $\mathrm{mx} /$ moodle/; el tour virtual de la UNAM-Centro de Enseñanza de Lenguas Extranjeras de México: http://ced.cele.unam. $\mathrm{mx} /$ formaus_biblioteca/sinicial/indice.html; y el manual para citas y trabajos académicos de la Universidad Federal de Rio Grande do Sul de Brasil: http://www.ufrgs.br/bibgeo/index. php?option =com_remository \&Itemid $=192 \&$ func $=$ startdown $\&$ id $=27$, entre otros.

[25] En el caso concreto de formación dirigida a profesoresinvestigadores, estas temáticas son las que generan mayor interés y distintas bibliotecas universitarias lo han detectado, por ejemplo, la Pontificia Universidad Católica de Valparaíso de Chile: http://biblioteca.ucv.cl/; la Universidad de La Sabana de Colombia: http://www.unisabana.edu.co/unidades/ cta/ruta-de-formacion-docente/componente-1-competencias-informacionales/; la Universidad de Costa Rica: http:// sibdi.ucr.ac.cr/infoencuentro.jsp; la Universidad de Aveiro de Portugal: http://www.ua.pt/sbidm/biblioteca/PageText. aspx?id=14497; y la Universidad de Extremadura: http:// biblioteca.unex.es/aprendizaje-e-investigacion/investigacion. html; y de Navarra de España: http://www.unav.edu/web/ biblioteca/formacion. También la formación ALFIN dirigida al personal de administración y servicio está presente en varias bibliotecas universitarias españolas.

[26] En cuanto a programas de ALFIN realizados desde bibliotecas virtuales destacamos los ejemplos de la Universidad Autónoma de San Luis de Potosí de México y su biblioteca digital Creativa: http://creativa.uaslp.mx/formacion_06.html, y de la biblioteca digital de la Universidad de Oriente de Venezuela: http://bibliotecadigital.udo.edu.ve/6gigantes/index.htm

[27] Desde la Pontificia Universidad Católica de Chile en su proceso formativo el test de inicio es una herramienta clave para guiar al estudiante en los niveles y cursos que debe realizar: http://bibliotecas.uc.cl/index2.php?option=com content\&view $=$ article\&id $=237$, este método se emplea tam- 
bién en las Universidades Juárez Autónoma de Tabasco de México: http://www.ruv.itesm.mx/convenio/tabasco/oas/ minf/eva_diagnostica/homedoc.htm y Universidad Panamericana sede Guadalajara de México: https://sites.google.com/ a/up.edu.mx/crai-up-induccion-y-formacion-continua/desarrollo-de-habilidades-informativas

[28] Desde la perspectiva de esta formación con didácticas más activas (práctica basada en la evidencia) sobresale el caso de la biblioteca de psicología de la Universidad de Sao Paulo de Brasil: http://www.ip.usp.br/portal/index. php?option=com_content\&view=article\&id=2909 \%3Aarquivos-para-download\&catid = 123\%3Acapacitacao-cursos-etreinamento\&Itemid $=142 \&$ lang $=p t$

[29] Un ejemplo de estas acciones de extensión desde ALFIN sería el trabajo que hace por ejemplo la Universidad de Huelva de España: http://www.uhu.es/biblioteca/formacion/ programa_2012-2013.html\#diez

[30] Un caso especial relacionado con esta lección es el de la Universidad Autónoma de Ciudad Juárez de México, que a través de sus Encuentros Internacionales de DHI iniciados en 1997, ha ido capacitando permanentemente a sus bibliotecarios en esta temática y generando espacios de benchmarking: http://bivir.uacj.mx/dhi/Eventos/Default.htm e intercambio permanente (coordinan la lista de discusión DHI México: http://mx.groups.yahoo.com/ group/dhi-uacj/?v=1\&t=searc $\mathrm{h} \& \mathrm{ch}=$ web \&pub $=$ groups $\& \mathrm{sec}=$ group $\& \mathrm{~s} \mid \mathrm{k}=1$, Recientemente ha desarrollado una Maestría, que ha permitido cualificar más a su personal formador en ALFIN: http://www.uacj.mx/ICSA/ humanidades/MGSI/Paginas/ default.aspx

[31] Finalmente, el Wiki ALFIN/Iberoamérica: http://alfiniberoamerica.wikispaces.com/ y el Mapa de Proyectos de ALFIN/Iberoamérica: http://bit.ly/9hu80u, permite visualizar toda esa producción ofreciendo un enfoque teórico-aplicado sobre ALFIN de cerca de 300 bibliotecas universitarias iberoamericanas.

\section{BIBLIOGRAFÍA}

Ahmad Sharif, M.N.; Kamaruddin Malik, M.; Alias, R.A.; Shamsul, S.; Hidayati Zakaria, N. (2004). Knowledge Management (KM). Framework for Representing Lessons Learned System for Communities of practice in institutions of higher learning. Malaysian Journal of Computer Science, Vol. 17 (1), 1-12.

Bartczak, S.M.; Blair, A.M.; Peachey, T.A.; Turner, J.M. (2010). KM Capability Assessment: A Qualitative Approach. Proceedings of the Southern Association for Information Systems Conference, Atlanta, GA USA March 26th-27th. http://sais. aisnet.org/2010/2010-SAIS\%20Proceedings/ Bartczak-et-al.pdf [Consultado: 21-6-2013]

Burley, D. L.; Pandit, G. (2008). Lesson learned: Organizational realities influence KMS implementation. VINE: The Journal of Information and Knowledge Management Systems, Vol. 38 (4).

Carvalho Dantas, C. Leite, J.L.; Soares de Lima, S.B.; Conceição Stipp, M.A. (2009). Teoría fundamentada en los datos - aspectos conceptuales y operacionales: metodología posible de ser aplicada en la investigación en enfermería. Rev Latino-am Enfermagem, Vol. 17 (4). http:// www.scielo.br/pdf/rlae/v17n4/es_21.pdf [Consultado: 21-6-2013]
Easterby-Smith, M.; Lyles, M. -Ed- (2003) The Blackwell Handbook of Organizational Learning and Knowledge Management. Australia: Blackwell Publishing. 676 p.

Freeze, R. D.; Kulkarni, U. (2007). Knowledge management capability: Defining knowledge assets. Journal of Knowledge Management, Vol. 11(6), 94-109. http://dx.doi. org/10.1108/13673270710832190

Gratch-Lindauer, B. (2004). The three Arenas of Information Literacy Assessment. Reference \& User Services Quarterly, Vol. 44 (2), 122-129. Pasadas Ureña, C. (2006). -Trad.-. Los tres ámbitos de evaluación de la alfabetización informacional. Anales de Documentación, (9), 69-81. http://revistas.um.es/analesdoc/article/download/1411/1461 [Consultado: 11-8-2010]

Milton, N. (2010). The lessons learned handbook: Practical approaches to learning from experience. London: Chandos Publishing. 192 p. http:// dx.doi.org/10.1533/9781780631929

Ontko, M. ; Williamson, S. ; Kemp, R. ; Haselkorn, M. (2007). An Examination of the Effectiveness of Lessons-Learned Reporting within the Humanitarian Sector. The Journal of Information Technology in Social Change. April.

Patton, M.Q. (2001). Evaluation, Knowledge Management, Best Practices, and High Quality Lessons Learned. American Journal of Evaluation, (22), 329-336. http://dx.doi. org/10.1177/109821400102200307 / http:// dx.doi.org/10.1016/S1098-2140(01)00147-3

Patton, M.Q. (2002). Qualitative research and evaluation methods (3rd ed.). Thousand Oaks, CA: Sage. 688 p.

Pinto, M. (2010). Design of the IL-HUMASS survey on information literacy in higher education: A self-assessment approach. Journal of Information Science, Vol. 36(1), 86-103. http://dx.doi. org/10.1177/0165551509351198

Pinto, M.; Doucet, A.V. (2007). An Educational Resource for Information Literacy in Higher Education: Visibility and Usability of the e-COMS Academic Portal. Scientometrics, Vol. 72 (2), 225-252. http://dx.doi.org/10.1007/s11192007-1725-9

Pinto, M.; Uribe-Tirado, A. (2011). Formación del bibliotecario como alfabetizador informacional. Anuario ThinkEPI, 2011, 13-21. http://eprints.rclis.org/ handle/10760/15790 [Consultado: 21-6-2013].

Pinto, M.; Uribe-Tirado, A. (2012). Las bibliotecas públicas híbridas en el marco de la Alfabetización Informacional. Revista Española de Documentación Científica, (monográfico). http:// dx.doi.org/10.3989/redc.2012.mono.980 [Consultado: 21-6-2013]

Pinto, M.; Sales, D.; Osorio, P. (2008). Biblioteca universitaria, CRAI y alfabetización informacional. Gijón: Ediciones TREA, 246 p.

Pinto, M.; Uribe-Tirado, A.; Gómez Díaz, R.; Cordón, J.A. (2011). La producción científica inter- 
nacional sobre competencias informacionales e informáticas: tendencias e interrelaciones. Información, Cultura y Sociedad, (25). http:// www.scielo.org.ar/pdf/ics/n25/n25a04.pdf [Consultado: 21-6-2013]

Pinto, M.; Fernández-Ramos, A.; Sánchez, G., Meneses, G. (2013). Information Competence of Doctoral Students in Information Science in Spain and Latin America: A Self-assessment. Journal of Academic Librarianship, Vol. 39 (2), 144-154. http://dx.doi.org/10.1016/j.acalib.2012.08.006

Roulston, K.; McClendon, V. Thomas, A.; Tuff, R.; Williams, G.; Healy, M. (2008). Developing reflective interviewers and reflexive researchers. Reflective Practice, Vol. 9 (3), 231-243. http:// dx.doi.org/10.1080/14623940802206958

Strauss, A.; Corbin, J. (2002). Bases de la investigación cualitativa. Técnicas y procedimientos para desarrollar una teoría fundamentada. Medellín: Editorial Universidad de Antioquia. 342 p.

Syed-Ikhsan, S.O.S.; Rowland, F. (2004). Knowledge management in a public organization: A study on the relationship between organizational elements and the performance of knowledge transfer. Journal of Knowledge Management, Vol. 8 (2), 95-111. http://dx.doi. org/10.1108/13673270410529145

Uribe-Tirado, A. (2004). Acceso, conocimiento y uso de las herramientas especializadas de internet entre la comunidad académica, científica, profesional y cultural de la Universidad de Antioquia. Especialización en gerencia de servicios de información. Escuela Interamericana de Bibliotecología. Universidad de Antioquia (Colombia) http://eprints.rclis.org/ handle/10760/6206\# . T1qhOIEaPIM [Consultado: 21-6-2013]
Uribe-Tirado, A. (2008). Diseño e implementación y evaluación de una propuesta formativa en alfabetización informacional mediante un ambiente virtual de aprendizaje a nivel universitario: caso Escuela Interamericana de Bibliotecología, Universidad de Antioquía. Maestría en Ingeniería, Línea Informática Educativa. Universidad EAFIT (Colombia). http://eprints.rclis.org/handle/10760/12606 [Consultado: 21-6-2013]

Uribe-Tirado, A.; Arroyave Palacio, M.; Ramírez Marín, G.J.; Pineda Gaviria, M.; Valderrama Muñoz, A.M.; Preciado, J.F. (2008). Acceso, conocimiento y uso de Internet en la universidad. Modelo de diagnóstico y caracterización: Caso Universidad de Antioquia. Medellín: Universidad de Antioquia / EIB-CICINF, 180 p. http://eprints. rclis.org/bitstream/10760/12543/1/Libro_Internet_Universidad_de_Antioquia.pdf [Consultado: 21-6-2013]

Uribe-Tirado, A. (2010). La Alfabetización Informacional en Iberoamérica. Una aproximación a su pasado, presente y futuro desde el análisis de la literatura publicada y los recursos web. IBERSID: revista de sistemas de información y documentación. Universidad de Zaragoza, 2010, 165-176. http://eprints.rclis.org/handle/10760/15060 [Consultado: 25-10-2011]

Wellman. J. (2007). Lessons Learned About Lessons Learned. Organization Development Journal, Vol. 25 (3). http://cmapspublic.ihmc.us/ri $\mathrm{d}=1221824070590 \_649857522$ 13181/ lessons $\% 20$ learned $\% 20$ about $\% 20$ lessons $\% 20$ leaned_wellman.pdf [Consultado: 21-6-2013]

Wheeldon, J.; Ahlberg, M.K. (2012). Visualizing Social Science Research. Maps, Methods \& Meaning. Los Angeles: Sage Publications. 224 p. 